\title{
Research and Implementation of Training Platform Based On Cloud Computer System
}

\author{
Yu Mingyi \\ Address: Chongqing College of Electronic Engineering, Chongqing, China \\ Email: 12399395 @qq.com
}

\begin{abstract}
Keywords: Cloud Computing; Computer Teaching; Training Platform
\end{abstract}
\begin{abstract}
The application of cloud computing technology in the network technology has changed the operation of computer training teaching pattern. Considering on the situation of computer training course, this paper put forward the operation cloud computing technology to improve the current computer training platform, and developed the corresponding computer training teaching management platform. Based on tracking and managing the computer training process, the system had the functions of the courseware management, the sharing of resources, the automatic generation of papers, the on-line assessment and so on. With the test, it is found that the function of the system is stable and can meet the needs of the computer training teaching.
\end{abstract}

\section{Introduction}

With the gradual deepening of teaching reform and the "Quality Project" full implementation of computer experiment and training teaching colleges and universities more and more attention, training teaching system based on cloud computing emerged. It can be daily teaching training colleges and universities to digitize and construction management, training teaching information technology for arranging training teaching management, efficient utilization of laboratory statistics, and so on. And the user is not required to run the application on a personal computer client (mobile phones and other devices, but the Internet running large-scale server clusters. The user data is not stored locally processed, but stored in the Internet data center. the data center management and maintenance of the normal operation of cloud computing services provided by the company responsible by them to ensure strong enough computing power and storage space large enough for the user to use at any time and any place , users can connect to the Internet at any terminal device. At the same time, the function of the user terminal will be greatly simplified in the cloud, and many complex functions will be transferred to the terminal behind the network up is complete.

\section{Cloud Computer System}

Cloud computer system called cloud systems, cloud computing background data center management and operation of the overall system. Architecture cloud system is relatively simple and it mainly consists of two parts: Cloud computing and cloud storage. That cloud computing distributed computing, database set up by the cluster computer services around the needs of different services provided through the transmission and distribution network data for the user; cloud storage is the same reason, the user's data is stored in the cloud computer, avoid using local storage resources, to use off-site and off-site storage purposes. Cloud computer refers to the use of personal computers and supercomputers completely different distributed architectures, virtualization technology by means of cloud computing, the integration of a plurality of relatively low-cost computing resources has made a powerful computing power and memory computer capabilities. Universities cloud computing technology to build a virtual training room and you can enhance the abilities of students and learning ability. 


\section{Features of Cloud Computer System}

High Availability and High Scalability. Cloud computer system itself is composed of a plurality of computer components, which can be a powerful air, contributing to Virtual Clusters. While the cluster, but also to meet the various separate computing units to work very well to avoid interference to other units, and after its recovery, configuration information can quickly be missed by other computing unit to deliver updates.

High Reliability. Cloud computing has a very complex network architecture and operational procedures, based on this, to cloud computing provides highly reliable, if multiple redundant sites are used, you can continue to improve its reliability.

Great Versatility. A cloud computing database can supply an unlimited number of computer users use data and information, the user has versatility. In addition, cloud computing for the needs of different users and different software, different construction applications, easy to use a variety of approaches.

Transparency and Pooling Resources. Cloud computing resource providers offer is transparent, and it is quite large, the user can always access any data without having to understand the internal structure of cloud computing services, as long as they need to understand the data can be. The so-called pooling is the cloud computing provider of data, integrated management of resources allocation, completely broken the boundaries and limitations of resources.

Desktop Backup and Disaster Recovery. Cloud computer system to automatically back up data of the original computer system in a virtual environment of cloud computing, data storage for information mainly discrete approach. Compared to traditional data recovery, data recovery cloud data is relatively simple, for the time required for recovery becomes shorter. Therefore, the cloud database for data backup is an important measure of computer network security protection. Effective data backup and update information on a regular basis, to avoid data loss, even if the data is changed, it can be restored based on the backup data.

\section{Key Technology Analysis of System Implementation}

Programming Model of Cloud Sharing System. Design Computer Practical Teaching Platform cloud computer is mainly used in B / S and C / S hybrid architecture model, using J2EE architecture program, a three-layer MVC design pattern, it can effectively reduce the pressure on the server system, improve the system use of effects. In operation, the students through the student's browser, to issue a request to the operating system, training, and sends the request to the Action Servlet processing system, the system process is finished, the request is sent to the system's central processor. Central processor based on the content of the corresponding request, further judgment and decided to send a request to the appropriate registration server, to ensure that the appropriate services in the server to complete processing.

WEB Related Technologies. In the system development and design process, it requires each running program needs to run in a safe working environment, in order to effectively ensure the safe operation of the program. Development of the system is mainly used in the standard Java technology architecture, student-side, learners can directly call the relevant procedures to achieve their own learning. As a result of APP related technologies, portability system is better for some small Java program, just a little change can be used in a system, to meet the different needs of system development, according to the same system development needs, mainly through the Struts2, Spring2, JDO, JavaScript, CSS and other technologies developed to meet the needs of system development.

\section{The Implementation of Computer Practical Teaching System Based on Cloud Computing}

Build A Cloud Platform Training Environment. In Computer Practical aspects of the operation, in order to meet the requirements of large-scale training of students, cloud operating platform environment need to set up six nodes. Specifically, the configuration of six working in Vmware 
Workstation virtual machine, the system managed to achieve the purpose of real-time manipulation, each virtual machine should be configured with a node, and communicating with the server. Among them, the need to set up a master node or node name of the node (Name Node), connectivity to the main server system, and the remaining five nodes as child nodes or data bus system (Date Node), in a specific configuration, this needs a few nodes are configured with the appropriate IP address assignment and host, the specific configuration shown in table 1.

Tab. 1 System node IP and configuration of host

\begin{tabular}{lcc}
\hline Node Type & IP Address analysis & Host Name \\
\hline Name node & 192.168 .1 .110 & Master \\
Date Node & 192.168 .1 .111 & Slaver1 \\
Date Node & 192.168 .1 .112 & Slaver2 \\
Date Node & 192.168 .1 .113 & Slaver3 \\
Date Node & 192.168 .1 .114 & Slaver4 \\
Date Node & 192.168 .1 .115 & Slaver5 \\
\hline
\end{tabular}

Building process based on cloud computing platform computer training is a more complex process, in the configuration of the process, the need to establish a system of SSH login password no password, and generates a key pair on each node of the system, to achieve management system, then the system will generate a key pair are replicated to the other six node system to ensure the system can work effectively.

Submit Business Achievement of Training Effect. Students after completing training actions related content, submit to enter management page, select and click on the appropriate training mission submitted a list of options, submitted its training mission. State outcomes, if you submit complete status display, indicating that the user completed the training project has been submitted, after submitting the information is complete, the students according to their own needs, one can generate paper, measuring their own learning, and the other aspect, after clicking you can browse the contents of the papers. If you submit a status display is not completed, the student can not complete the follow-up of related operations

Achievement of Software Testing Services. Software testing system based on the corresponding specifications of the system in actual testing session, mainly through the black box testing method, specific hybrid model of cloud computing computer training platform B / S, C / S-based client systems through a browser sent to the relevant source server performance evaluation, analysis server during operation stability, security, fault tolerance test, and the test data were analyzed [10]. Through the training system operating functions, papers generation, evaluation function, students and teachers of sign-on feature, broadcast, visualization experiment, program debugging function, courseware upload and download functions related functions for testing found that stable operation of the system, the system can meet the job requirements.

\section{Problems and Improvement Method of Cloud Computer System in Training Platform Construction Management}

Training Equipment Hardware Configuration Problem. The most basic problem is the computer hardware, hardware upgrading training base configuration issues, cloud computing platform in the role of training can be improved. Computer hardware system includes servers, storage devices, and other hardware-related network equipment. Measures to improve the cloud systems hardware devices are: high-performance servers and high-bandwidth combine to provide rental services in accordance with the needs of cloud computer users should find their own problems, hands update computer hardware, training and services platform based on the acquisition of the necessary training equipment hardware, update the host server and cloud.

The Operating Failures of Cloud System Training Platform User. Computer training platform in the cloud, users are most like their own uninstall the software, modify system settings and services, causing the computer speed is affected. In addition, some users looking for professionals to reinstall the system, but due to lack of user knowledge cloud systems inevitably 
deceived, the consequences could be disastrous. To address this issue, cloud computer solution is: rational use of data virtualization technology, which can be based on different categories, according to the user's will build their own desktop systems, depending on the experiment needs of system software and application software, generate different operating systems and processing methods. Its installation and use of the same physical host, but it saves a lot of time and energy, and to use its advantages can be shared by multiple users simultaneously using the participants, although modified virtual desktops in the cloud host reboot still be retained, unless the administrator were recovered primary mirror operation to recover after the restart of the virtual desktop is pristine condition.

Control of Virus. Students in the classroom using the U disk can cause the virus spread rapidly, because the host and personal training room compared to the host more susceptible to the virus and spread the virus. And the above-mentioned issues, the face of the virus must be prepared in advance prevention, which is currently the best method is to use advanced data virtualization technology. The user data virtualization, virtual desktops can provide computer users when viruses and malicious Trojans invasion, even if the current computer system damage, but only need to restart the computer, these virtual desktop system will be restored.

Coordination of Demand and Management. Teaching cloud reality computer system training platform, due to the large number of students, and the specialty and age, different, which means that every time you use cloud computer system training platform, we need to replace the artificial different systems and procedures, not only waste time learning and teachers teaching time, but also on cloud computer system training platform cause some damage. To address this issue, cloud computer also proposed solutions: the same use of virtualization technology to build a variety of content-rich virtual application pool, in the training process, should the need to temporarily use other applications and you only need to recover the original virtual master image, Add the required applications and deploy them.

Heavy Management Workload. In the original configuration of the room, form a problem of multi-room, multi-device and operating personnel shortage, which is due to the high frequency of use of students and demand, often resulting in a significant increase in the workload of the operator. To address this issue, cloud computer also proposed solutions: the same is the use of virtual technology cloud computer system, only one device, simple operation by an operator and you can achieve the desktop cloud application delivery, change and recover, reducing workload while meeting the diverse needs of students.

\section{Conclusion:}

In summary, university training platform after the introduction of cloud computer system, not only to provide students with a rich learning content, the actual operating level of the students has also been greatly improved. It implements a staff training or even multiple virtual servers, but also changed the traditional training situation not been widely used, and all aspects of saving the cost of investment, in terms of administrators, but also through virtual technology greatly reduces its work the amount. Cloud computer as a leading technology of integration resources, although there is no unified technical standards and implementation, it still needs to take full advantage of it in college training classroom.

\section{References}

[1] Ye Feng: Fujian Computer, Vol. 2 (2015) No 53, p.25-26

[2] Jin Yongqi: Hydropower Electromechanical Technology, Vol. 10 (2015) No 27, p.74-76

[3] Wu Lei: Jianghan University, Vol. 5 (2015) No 33, p.11-14

[4] Wang Xuerong: Computer Application, Vol. 3 (2010) No33, p.121-124

[5] Liang Jinxiong: Value Engineering, Vol. 13 (2011) No25, p.58-63 